\title{
Proteomic Insights into the Hidden World of Phloem Sap Feeding
}

\author{
Sohail Ahmad Khan Rao, James C. Carolan, and Thomas L. Wilkinson
}

\begin{abstract}
The physical interface between a phloem-feeding insect and its host plant is a single cell buried deep within the plant tissue. As such, the molecular interactions between these notorious agricultural pests and the crop plants upon which they feed are difficult to study. 'Omic' technologies have proved crucial in revealing some of the fascinating detail of the molecular interplay between these partners. Here we review the role of proteomics in identifying putative components of the secreted saliva of phloem-feeding insects, particularly aphids, and discuss the limited knowledge concerning the function of these proteins.
\end{abstract}

\section{Overview}

Phloem feeding insects represent a guild of agricultural pests that are notoriously difficult to study and even harder to control (van Emden and Harrington 2007). Much of the problem lies in the location of the feeding site since the physical interface between the insect and plant is a single sieve element cell within the phloem bundle buried deep in the leaf. As a consequence, ingestion of the diet cannot be observed directly as is possible in most chewing insects. Several approaches have been developed over the last few decades to address these issues and some notable achievements include the electrical penetration graph and the use of non-persistent plant viruses to determine the sequence of feeding behaviours between plant surface penetration and phloem sap ingestion (see Powell et al. 2006 for full review).

\footnotetext{
S.A.K. Rao

Centre of Agricultural Biochemistry and Biotechnology (CABB), University of Agriculture,

Faisalabad 38040, Pakistan

e-mail: srao@uaf.edu.pk

J.C. Carolan

Department of Biology, Maynooth University, Maynooth, County Kildare, Ireland e-mail: james.carolan@nuim.ie

T.L. Wilkinson $(\bowtie)$

UCD School of Biology and Environmental Science, University College Dublin,

Belfield, Dublin 4, Ireland

e-mail: tom.wilkinson@ucd.ie
} 
However much of the mechanistic detail involved in the location, acquisition and preparation of the feeding site remain obscure.

Advances in 'omics' technologies have improved the identification of putative genes and proteins with a direct involvement in the insect-plant interaction. Here, we review advances made by proteomic technologies in understanding the molecular interplay between phloem-feeding insects and plants, focussing on the proteins that have been identified as being secreted in the saliva. Much of the evidence comes from studies involving aphids, since this group of insects has extensive genomic and, to a lesser extent, proteomic resources available in the public domain coupled with a long history of agronomic research aimed at improving control strategies.

\section{Feeding Behaviour}

Phloem-feeding insects such as aphids, whiteflies and leafhoppers cause direct damage by removing photoassimilates and by the transmission of a variety of plant viruses. Host plant location and acceptance involves many steps that must occur in the correct sequence before feeding can commence. Initial plant recognition is mediated by the antennae and the mouthparts. Aphid antennae bear many sensilla which are used in chemoreception and the perception of the leaf surface (Bromley and Anderson 1982) and tactile receptors on the tip of the proboscis respond to contact and surface texture and enable aphids to detect the contours of leaf veins, their preferred feeding site (Tjallingii 1978; Powell et al. 2006).

Once a plant has been accepted, the aphid will settle and initiate penetration to the sieve element. Aphids feed from a single phloem cell within the sieve element and can continuously imbibe phloem sap for prolonged periods (Tjallingii 1995). The mouthparts are modified as piercing stylets formed by the paired mandibles and maxillae (Pollard 1973) that come together to form two distinct channels: (1) a salivary canal that transports saliva into the plant and (2) a food canal, through which phloem sap is ingested. The food canal has a larger diameter $(0.7 \mu \mathrm{m})$ than the salivary canal $(0.3 \mu \mathrm{m})$ (Ponsen 1987). Plant penetration can be monitored using the electrical penetration graph (EPG; McLean and Kinsey 1965; Tjallingii 2006) that allows the recording of signal waveforms reflecting different insect activities and locations of the stylet tips. Perhaps unsurprisingly there is a direct relationship between the length of the stylets and the depth of the phloem tissue within the host plant, but despite differences in insect size and the internal architecture of the host plant the mechanism of penetration appears to be similar across aphid groups - the stylets move intercellularly along and within cell walls without directly passing through any cell, to form a convoluted stylet track (Fig. 1). However, the aphid appears to taste and reject the contents of many cells as the stylet tips journey towards the sieve element (Tjallingii and Esch 1993). 


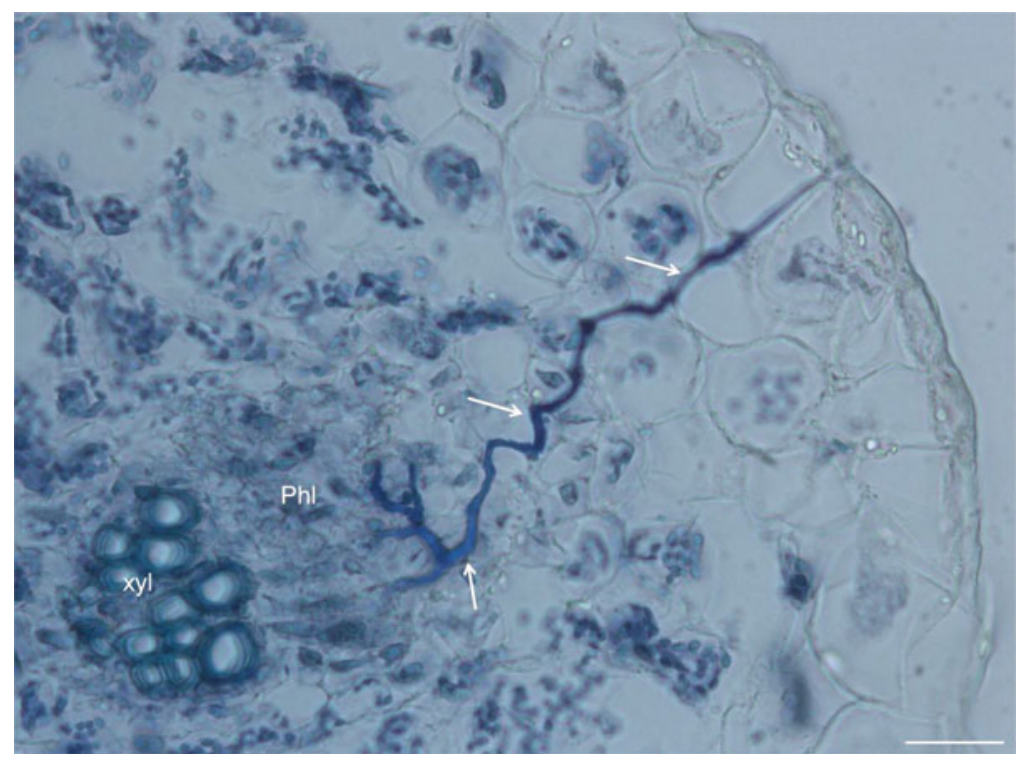

Fig. 1 Hand-cut section of broad bean Vicia faba cv. The Sutton showing salivary sheath remaining in the plant following feeding by the pea aphid Acyrthosiphon pisum. Phl phloem, xyl xylem, arrows sheath material. Scale bar $=100 \mu \mathrm{m}$

\section{Recognition of the Sieve Tube}

The ability to detect and find sieve tubes suggests that phloem feeding insects receive cues for sieve-tube recognition along the stylet route perhaps by sampling cells. Specific conditions such as $\mathrm{pH}$, sugar species and concentration, viscosity, and oxygen pressure may enable sieve-tube detection (Hewer et al. 2010). In early experiments, sucrose was determined as the most suitable carbohydrate substrate for aphid growth and reproduction, leading to the suggestion that sucrose is an important cue for aphid orientation (Auclair 1963, 1969; Mittler and Dadd 1964). However, recent experiments using artificial choice-chamber systems have suggested that $\mathrm{pH}$ is also an important orientation cue used by aphids to locate the sieve element (Hewer et al. 2010). Different aphid species (Megoura viciae, Myzus persicae, Rhopalosiphum padi and Macrosiphum euphorbiae) showed a significant preference for sucrose at concentrations of $15 \%$ and $\mathrm{pH} 7$ (over a test range of $\mathrm{pH} 5-8$ ) that matches the composition of the sieve-tube sap of their host plants (Hewer et al. 2010). However, further studies are warranted to determine the precise navigational cues employed by aphids and other phloem-feeders since this is an obvious potential target for disrupting feeding behaviour. 


\section{Aphid Salivation}

The secretion of saliva prior to and during phloem feeding has attracted significant attention. Two types of saliva are secreted during feeding - gelling saliva and watery saliva (Miles 1999; Cherqui and Tjallingii 2000). Prior to stylet insertion, the aphid secretes a small amount of gelling saliva onto the plant surface. This is termed the salivary flange and hardens almost immediately, perhaps upon contact with oxygen in the air. The aphid inserts its stylet through this gel and begins to probe into the plant. An individual aphid may make several probes over an extended period of time before accepting a sieve element for sustained ingestion. As the stylets progress through the plant gelling saliva is continuously secreted which hardens to form a rigid, protective sheath around the stylet. The so-called salivary sheath remains in the plant after stylet withdrawal irrespective of whether the aphid has successfully acquired a sieve element or not (see Fig. 1). During probing activities, very small amounts of watery saliva are discharged when parenchymal cells are briefly punctured, followed by ingestion of minute amounts of cell sap, before any sieve elements are punctured (Prado and Tjallingii 1994; Martin et al. 1997). The frequency of the brief cell punctures (referred to as 'potential drops' in EPG traces) often increases immediately before the onset of sustained ingestion, again suggesting that the aphid is obtaining some kind of navigational cue from the cell contents. Once the sieve element is located the gelling saliva forms a seal around the site of insertion. The secretion of gelling saliva ceases at this point and the aphid begins to secrete watery saliva. The transition between the secretion of gelling and watery saliva appears to be instantaneous but the mechanism controlling the switch in consistency and perhaps composition is unclear. The ingestion of phloem sap into the food canal is resisted initially and watery saliva is released into the contents of the sieve tube for approximately 5 min (Miles 1999). Thereafter, during ingestion, there is a continual secretion of watery saliva but, since the end of the salivary canal is a short distance behind the tip of the stylets, the saliva is ingested with the sieve tube sap under positive turgor pressure (Tjallingii 1995). Regulation of phloem sap intake is thought to be controlled by the precibarial valve at the opening of the anterior gut (Pollard 1973), but again this aspect of aphid feeding is poorly understood.

\section{Composition of Aphid Saliva}

From the previous discussion of the mechanistic aspects of aphid feeding it is clear that the salivary secretions play a crucial role in the aphid-plant interaction, not least because they represent a significant investment of resources by the insect. However, before realistic hypotheses concerning the function of salivary secretions can be formulated, a thorough understanding of the composition of the different types of saliva is required, and it is here that modern analytical techniques, particularly proteomics, have had a significant impact. 
Aphid saliva is an aqueous solution containing amino acids, haemolymph components (including proteins, see below) and proteins derived from the salivary glands (Miles 1999). Early studies in the literature adopted substrate-specific enzyme assays to determine bioactive components of saliva, but these are limited since they can only detect, by their very nature, those proteins that interact with the substrate. Nevertheless, these methods provided early recognition of the importance of bioactive molecules in the aphid-plant interaction. The salivary components detected by these methods can be divided into two broad categories: (i) hydrolases (pectinases, cellulases, oligosaccharases); and (ii) oxidation/reduction enzymes (phenol oxidase and peroxidases) (Miles 1999; Campbell and Dreyer 1985, 1990; Peng and Miles 1988; Madhusudhan and Miles 1998). The roles of most of these enzymes during aphid penetration and feeding are not well understood. The salivary sheath apparently contains proteins with active sulphydryl groups and it exhibits enzyme activity, including phenoloxidases and peroxidases, while, in addition, oxidases and pectinases have been reported in the watery saliva (Miles 1999). Aphids are able to access the chemical nature of the matrix polysaccharides in the cell wall and cell components of plants and would seem to do so by action of the hydrolytic enzymes that occur in the saliva (Miles 1999).

In the last decade our understanding of the complexities of aphid saliva has increased with the use of mass spectrometry and proteomics to identify salivary proteins. These techniques provide positive identities based on sequence homology with publicly available databases, and can detect novel proteins. The approach is therefore more comprehensive since it does not rely on a single substrate to detect the presence of a bioactive protein. However, in the absence of species-specific sequence information (either as genomic or transcriptomic data) only highly conserved proteins can be detected. Significant advances in the ability to identify aphid salivary proteins was achieved following the publication of the complete genome sequence of the pea aphid Acyrthosiphon pisum (International Aphid Genomics Consortium 2010) and further advances can be expected as more genomic resources become available for other phloem-feeding insects.

A detailed proteomic analysis of saliva secreted by the pea aphid Acyrthosiphon pisum showed the presence of nine proteins following GE-LC-MS/MS and LC-MS/ MS, with reference to expressed sequence tags (EST) and genomic sequence data for A. pisum (Carolan et al. 2009). Four proteins were identified by sequence homology: an M2 metalloprotease (a homolog of angiotensin-converting enzyme); an M1 zincdependant metalloprotease; a glucose-methanol-choline (GMC)-oxidoreductase; and a homolog to regucalcin (a putative calcium-binding protein). The other five proteins were not homologous to any previously described sequence and included an abundant salivary protein (ACYPI009881, see below) with a putative role in the formation of the salivary sheath (based on its amino acid composition). The metalloproteases and regucalcin were predicted to be directly involved in maintenance of sustained feeding through the inactivation of plant protein defences and inhibition of calcium-mediated occlusion of phloem sieve elements, respectively, and the oxidoreductase may promote gelling of the sheath protein or mediate oxidative detoxification of plant allelochemicals (Carolan et al. 2009). 
A multi-approach experiment based on both in-solution and in-gel (after 2D gel electrophoresis) protein digestion and complementary mass spectrometry techniques was used to investigate the salivary proteome of Myzus persicae (Harmel et al. 2008). Some proteins were identified with a known function in other insects, while others were related to aphid expressed sequence tag (EST) sequences from specific tissue locations (i.e. head and/or salivary glands).

The secreted saliva of the vetch aphid Megoura viciae (Will et al. 2007) comprised at least 29 proteins ranging in weight from 20 to $170 \mathrm{kDa}$ when analysed using one dimensional gel electrophoresis. Although no protein identities were obtained, a conformational change in two proteins at 40 and $43 \mathrm{kDa}$ when the gel was incubated in the presence of free calcium suggests that these proteins may play an important role in preventing formation of protein plugs (so-called forisomes) in Fabaceae (Will et al. 2007; see Will et al. 2012 for review). Interestingly, these proteins have a similar molecular weight to the regucalcin identified in the saliva of $A$. pisum (Carolan et al. 2009).

As our understanding of the detailed composition of aphid saliva increases, comparative analysis of saliva from different species or between 'biotypes' or clones of the same species is becoming possible. These studies have an applied implication since they reveal potential targets in pest species that might have an important role in future control strategies, but they can also reveal evolutionary links between feeding strategies, such as polyphagy and monophagy, and host plant choice including host plant alteration during the insect life cycle. As an example, a recent study (Rao et al. 2013) of secreted saliva from aphids that feed on cereals (colloquially referred to as 'cereal aphids', although the species concerned are not necessarily close phylogenetically) determined only three individual proteins that were also detected in the secreted salivary proteomes of A. pisum (Carolan et al. 2009, 2011) and $M$. persicae (Harmel et al. 2008) which feed on dicotyledonous plants. These common proteins (two paralogues from the GMC-oxidoreductase family referred to as glucose dehydrogenase or GLD, and the novel protein ACYPI009881) are both implicated in the formation of the salivary sheath and are discussed in more detail below.

The salivary sheath is a crucial structure common to a wide range of sap-feeding insects, including aphids, whiteflies and planthoppers (see Fig. 1). The abundance and amino-acid composition of the ACYPI009881 protein (Carolan et al. 2009) suggests that it may contribute to the sheath saliva. The conserved nature of this protein is significant given that the salivary sheath plays an important role in masking the presence of feeding aphids from plant defences, including preventing leakage of sieve element contents into the apoplast, a known trigger of plant defences (Tjallingii 2006; Will and van Bel 2006; Will et al. 2007). The hypothetical protein ACYPI009881 (referred to as sheath protein or SHP) was common to the cereal aphids Sitobion avenae and Metopolophium dirhodum and has previously been identified from the secreted saliva and salivary gland of A. pisum (Carolan et al. 2009, 2011) indicating that SHP may be common to a wide variety of aphid species. Immunoblotting using antibodies raised against SHP confirmed the presence of the protein in both secreted saliva and salivary gland extracts from $S$. avenae and M. dirhodum. In addition, SHP was localized to specific cell types within the salivary gland (Rao et al. 2013). 
The other common proteins belong to the GMC-oxidoreductase family and these are the most frequently reported bioactive proteins in studies of aphid saliva detected either by substrate-specific assays (Madhusudhan and Miles 1998) or by direct identification using mass spectrometry (Harmel et al. 2008; Carolan et al. 2009; Nicholson et al. 2012; Rao et al. 2013; Vandermorten et al. 2014; Nicholson and Puterka 2014; see Table 1). GMC oxidoreductase in insect saliva in general has been implicated in the modification of plant defence mechanisms (Eichenseer et al. 1999; Musser et al. 2002; 2005), and in aphids specifically is speculated to be involved in the detoxification of noxious phytochemicals and in promoting the gelling of sheath saliva by enhancing disulphide bridge formation (Miles and Oertli 1993). GMCoxidoreductase has been detected in the secreted saliva, but the protein does not originate in the salivary gland (Rao et al. 2013) and is most likely imported from the haemolymph. A model indicating the putative origin and routes of secretion of two common salivary proteins is suggested in Fig. 2. Interestingly, glucose dehydrogenase, another member of the GMC-oxidoreductase family, was also detected in the saliva of Diuraphis noxia and Schizaphis graminum, two pests of cereals in the USA that cause phytotoxic lesions following feeding (Nicholson et al. 2012; Nicholson and Puterka 2014). Glucose dehydrogenase was the only significant protein in the watery saliva of $S$. graminum in common with other aphid salivary proteomes (Nicholson and Puterka 2014), but analysis of the saliva from virulent and avirulent strains of this notorious pest suggested that the protein composition of the saliva might be an important factor in determining host plant responses to aphid feeding.

Evidence for the involvement of a third party in the composition of aphid saliva is slowly emerging. The presence in the saliva of the chaperonin GroEL with sequence homology matching to the primary endosymbiotic bacteria Buchnera aphidicola (Filichkin et al. 1997; Vandermorten et al. 2014; Chaudhary et al. 2014) suggests an intriguing role for the bacteria in supplying molecular patterns that can be recognized by plant defenses. A detailed analysis following artificial introduction of GroEL via either direct application or transfection demonstrated recognition by and activation of the plant immune response, and a negative impact on the performance of feeding aphids (Chaudhary et al. 2014). However, the direct involvement of Buchnera-derived GroEL in the priming of plant defenses requires further confirmation, and no study has detected a Buchnera-derived protein in planta.

All the secreted salivary proteomes reported to date have been obtained by analysis of proteins recovered from artificial liquid diets held between layers of stretched membrane. The recovery of sufficient quantities of secreted protein for analysis by mass spectrometry requires the concentration of large volumes of diet and multiple collections that are pooled into a single sample. There are obvious technical difficulties associated with this technique, including awareness of contamination and false positives, but the major drawback concerns the biological significance of the protein libraries. The diet system is by its very nature artificial and requires a phloem-feeding insect to actively rather than passively ingest (as is normally the case), and there is no consensus on the most appropriate composition of the diet from which to retrieve salivary proteins. Nevertheless, the identification of secreted proteins is a valuable first step towards more detailed functional studies, including the identification of salivary proteins in planta, which will provide a deeper 


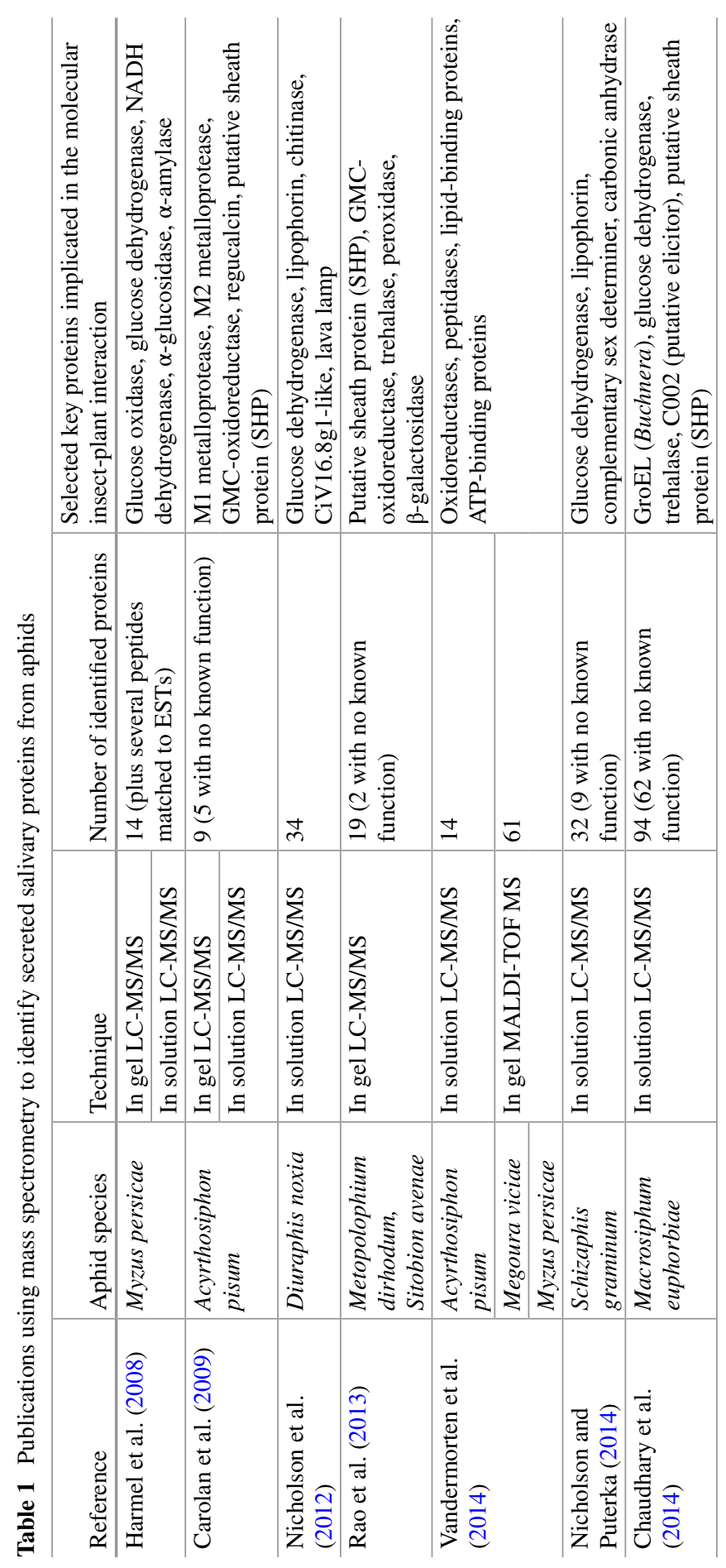




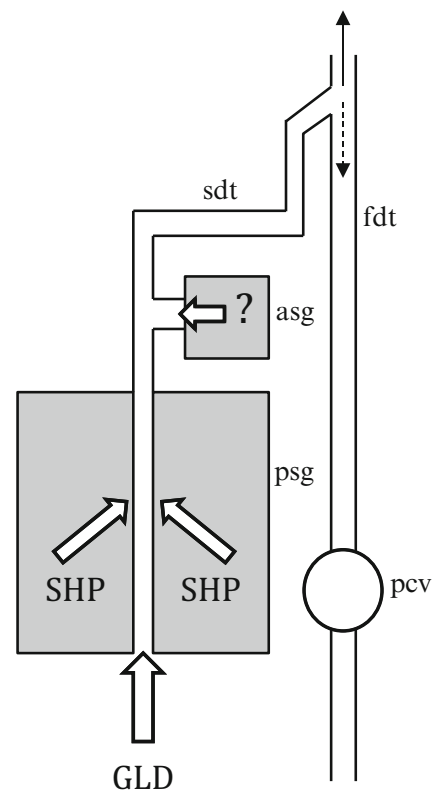

Fig. 2 Schematic representation of the origin and secretion of salivary proteins by phloem-feeding aphids. Only one half of the paired salivary gland is shown (shaded). Blocked arrows indicate origin of salivary proteins; line arrows indicate direction of saliva flow with solid arrow if precibarial valve is closed (salivation into the plant) and dashed arrow if pre-cibarial valve is open (during ingestion). The contribution of the accessory gland to the secreted saliva remains unclear. SHP putative sheath protein (ACYPI009881), GLD glucose dehydrogenase, asg accessory salivary gland, $p s g$ principal salivary gland, $f d t$ food duct, $s d t$ salivary duct, $p c v$ pre-cibarial valve

understanding of the role of the saliva in the insect-plant interaction. The limited numbers of publications that have adopted mass spectrometry to identify secreted salivary proteins from aphids are detailed in Table 1.

\section{Importance of the Salivary Gland}

The origin of the secreted saliva (i.e. the material of aphid origin that enters the plant prior to and during ingestion) is the salivary gland. In aphids, the glands are paired and consist of two principal glands and two accessory glands located between the head and pro-thorax. A large, bi-lobed principal gland joins with a smaller accessory gland to form one half of the gland and the two sides unite through the common salivary duct that leads to the mouthparts (Ponsen 1972). A similar distinction between accessory and principal glands is seen in other phloem feeding insects, although the principal gland may be structurally more complicated. As an example, 
the principal glands of leafhoppers have multiple lobes each consisting of distinct groups of follicle cells (Sogawa 1965). The distinction between principal and accessory gland is largely based on size and morphology, with no direct evidence of the functional significance of each tissue. However, the accessory gland has been implicated as the conduit for plant virus accession to the saliva (Gildow and Gray 1993), perhaps in conjunction with the influx of water, and consequently further investigation is warranted. The importance of the principal gland in the production of at least some of the salivary proteins has already been highlighted, but it also appears to act as a conduit for the introduction of components from the haemolymph that could be derived from a variety of different tissues, including the fat body. As a consequence, putative libraries of salivary proteins derived from analysis of the salivary gland alone could be misleading.

A dual transcriptomic-proteomic analysis of the salivary gland of A. pisum used a bioinformatics approach to select a suite of identified proteins that had the potential to be included in the secreted proteome (Carolan et al. 2011). Amongst these were a group of so-called effector proteins (i.e. proteins that alter host plant cell structure or function) based on their homology or similarity to pathogenesis- or parasitism-related effector proteins secreted by other plant pathogens, particularly plant pathogenic nematodes. In particular, proteins in aphid saliva could have homologous or analogous functions to the giant cell modifying proteins of plant parasitic nematodes (Carolan et al. 2011).

\section{Conclusions}

In contrast to feeding by leaf chewing insects, the damage to plants caused by phloem feeding insects such as aphids is largely hidden from view and difficult to study. Consequently, modern approaches to combat phloem feeding pests have lagged behind advances in targeting leaf chewing insects despite their economic impact and predicted increasing global importance. The damage inflicted by aphids can be directly related to their ability to bypass and/or overcome host plant defences and a detailed mechanistic understanding of the process at a molecular level could lead to novel control strategies, e.g. through the use of highly selective RNA interference (RNAi; Pitino et al. 2011). Aphids can puncture and feed from plants for long periods without inducing a plant wound response and it has long been suspected that components of aphid saliva play a critical role in preventing blockage of the feeding site and/or detection of the mouthparts by the plant (Miles 1999; Will and van Bel 2006), but a detailed understanding of the composition of aphid saliva has only recently emerged. On a wider scale, the ever increasing human population requires a parallel increase in crop yields, yet increasing concerns about environmental safety have led to more stringent restrictions in the use of insecticides particularly in Europe. In addition, insects are renowned for developing resistance against insecticides, which further reduces the effectiveness of this control measure in the long term. For these reasons it is of importance to understand the molecular mechanisms that mediate the interaction between aphids and their host plants so 
that new methodologies can be developed that reduce agrochemical inputs. With the availability of more genomic and proteomic resources, the field will expand rapidly and will have increasing relevance to other phloem-feeding insects such as planthoppers and whiteflies.

\section{References}

Auclair, J. L. (1963). Aphid feeding and nutrition. Annual Review of Entomology, 8, 439.

Auclair, J. L. (1969). Nutrition of plant-sucking insects on chemically defined diets. Entomologia Experimentalis Et Applicata, 12, 623.

Bromley, A. K., \& Anderson, M. (1982). An electrophysiological study of olfaction in the aphid Nasonovia ribis-nigri. Entomologia Experimentalis et Applicata, 32, 101-110.

Campbell, D. C., \& Dreyer, D. L. (1985). Host-plant resistance of sorghum - Differential hydrolysis of sorghum pectic substances by polysacharases of greenbug biotypes (Schizaphis graminum Homoptera, Aphididae). Archives of Insect Biochemistry and Physiology, 2, 203-215.

Campbell, D. C., \& Dreyer, D. L. (1990). The role of plant matrix polysaccharides in aphid-plant interactions. In R. K. Campbell \& R. D. Eikenbary (Eds.), Aphid-plant genotype interactions (pp. 149-169). Amsterdam: Elsevier.

Carolan, J. C., Fitzroy, C. I., Ashton, P. D., Douglas, A. E., \& Wilkinson, T. L. (2009). The secreted salivary proteome of the pea aphid Acyrthosiphon pisum characterised by mass spectrometry. Proteomics, 9, 2457-2467.

Carolan, J. C., Caragea, D., Reardon, K. T., Mutti, N. S., Dittmer, N., Pappan, K., Cui, F., Castaneto, M., Poulain, J., Dossat, C., Tagu, D., Reese, J. C., Reeck, G. R., Wilkinson, T. L., \& Edwards, O. R. (2011). Predicted effector molecules in the salivary secretome of the pea aphid (Acyrthosiphon pisum): a dual transcriptomic/proteomic approach. Journal of Proteome Research, 10, 1505-1518.

Chaudhary, R., Atamian, H. S., Shen, Z., Briggs, S. P., \& Kaloshian, I. (2014). GroEL from the endosymbiont Buchnera aphidicola betrays the aphid by triggering plant defense. Proceedings of the National Academy of Sciences of the United States of America, 111, 8919-8924.

Cherqui, A., \& Tjallingii, W. F. (2000). Salivary proteins of aphids, a pilot study on identification, separation and immunolocalisation. Journal of Insect Physiology, 46, 1177-1186.

Eichenseer, H., Mathews, M. C., Bi, J. L., Murphy, J. B., \& Felton, G. W. (1999). Salivary glucose oxidase: Multifunctional roles for helicoverpa zea? Archives of Insect Biochemistry and Physiology, 42, 99-109.

Filichkin, S. A., Brumfield, S., Filichkin, T. P., \& Young, M. J. (1997). In vitro interactions of the aphid endosymbiont SymL chaperonin with barley yellow dwarf virus. Journal of Virology, 71, 569-577.

Gildow, F. E., \& Gray, S. M. (1993). The aphid salivary gland basal lamina as a selective barrier associated with vector-specific transmission of barley yellow dwarf luteoviruses. Phytopathology, 83, 1293-1302.

Harmel, N., Letocart, E., Cherqui, A., Giordanengo, P., Mazzucchelli, G., Guillonneau, F., DE Pauw, E., Haubruge, E., \& Francis, F. (2008). Identification of aphid salivary proteins: A proteomic investigation of Myzus persicae. Insect Molecular Biology, 17, 165-174.

Hewer, A., Will, T., \& van Bel, A. J. (2010). Plant cues for aphid navigation in vascular tissues. The Journal of Experimental Biology, 213, 4030-4042.

International Aphid Genomics Consortium. (2010). Genome sequence of the pea aphid Acyrthosiphon pisum. PLoS Biology, 8(2), e1000313. doi:10.1371/journal.pbio.1000313.

Madhusudhan, V. V., \& Miles, P. W. (1998). Mobility of salivary components as a possible reason for differences in the responses of alfalfa to the spotted alfalfa aphid and pea aphid. Entomologia Experimentalis Et Applicata, 86, 25-39. 
Martin, B., Collar, J. L., Tjallingii, W. F., \& Fereres, A. (1997). Intracellular ingestion and salivation by aphids may cause the acquisition and inoculation of non-persistently transmitted plant viruses. The Journal of General Virology, 78(Pt 10), 2701-2705.

Mclean, D. L., \& Kinsey, M. G. (1965). Identification of electrically recorded curve patterns associated with aphid salivation and ingestion. Nature, 205, 1130-1131.

Miles, P. W. (1999). Aphid saliva. Biological Reviews, 74, 41-85.

Miles, P. W., \& Oertli, J. J. (1993). The significance of antioxidants in the aphid-plant interaction The redox hypothesis. Entomologia Experimentalis Et Applicata, 67, 275-283.

Mittler, T. E., \& Dadd, R. H. (1964). Gustatory discrimination between liquids by the aphid Myzus persicae (Sulzer). Entomologia Experimentalis Et Applicata, 7, 315-328.

Musser, R. O., Hum-Musser, S. M., Eichenseer, H., Peiffer, M., Ervin, G., Murphy, J. B., \& Felton, G. W. (2002). Herbivory: caterpillar saliva beats plant defences. Nature, 416, 599-600.

Musser, R. O., Cipollini, D. F., Hum-Musser, S. M., Williams, S. A., Brown, J. K., \& Felton, G. W. (2005). Evidence that the caterpillar salivary enzyme glucose oxidase provides herbivore offense in solanaceous plants. Archives of Insect Biochemistry and Physiology, 58, 128-137.

Nicholson, S. J., \& Puterka, G. J. (2014). Variation in the salivary proteomes of differentially virulent greenbug (Schizaphis graminum Rondani) biotypes. Journal of Proteomics, 105, 186-203.

Nicholson, S. J., Hartson, S. D., \& Puterka, G. J. (2012). Proteomic analysis of secreted saliva from Russian Wheat Aphid (Diuraphis noxia Kurd.) biotypes that differ in virulence to wheat. Journal of Proteomics, 75, 2252-2268.

Peng, Z., \& Miles, P. W. (1988). Studies on the salivary physiology of plant bugs - Function of the catechol oxidase of the rose aphid. Journal of Insect Physiology, 34, 1027-1033.

Pitino, M., Coleman, A. D., Maffei, M. E., Ridout, C. J., \& Hogenhout, S. A. (2011). Silencing of aphid genes by dsRNA feeding from plants. PLoS One, 6(10), e25709. doi:10.1371/journal. pone.0025709.

Pollard, D. G. (1973). Plant penetration by feeding aphids (Hemiptera, Aphidoidea). Bulletin of Entomological Research, 62, 631-714.

Ponsen, M. B. (1972). The site of potato leaf roll virus multiplication in its vector Myzus persicae, an anatomical study. Meded Landbouwhogesch Wageningen, 16, 1-147.

Ponsen, M. B. (1987). Alimentary tract. In A. K. Minks \& P. Harrewijn (Eds.), Aphids, their biology, natural enemies and control (World crop pests, Vol. 2A, pp. 79-96). Amsterdam: Elsevier.

Powell, G., Tosh, C. R., \& Hardie, J. (2006). Host plant selection by aphids: Behavioral, evolutionary, and applied perspectives. Annual Review of Entomology, 51, 309-330.

Prado, E., \& Tjallingii, W. F. (1994). Aphid activities during sieve element punctures. Entomologia Experimentalis Et Applicata, 72, 157-165.

Rao, S. A. K., Carolan, J. C., \& Wilkinson, T. L. (2013). Proteomic profiling of cereal aphid saliva reveals both ubiquitous and adaptive secreted proteins. PLoS One, 8(2), e57413. doi:10.1371/ journal.pone.0057413.

Sogawa, K. (1965). Studies on the salivary glands of rice plant leafhoppers. I Morphology and histology. Japanese Journal of Applied Entomology and Zoology, 9, 275-304.

Tjallingii, W. F. (1978). Electronic recording of penetration behavior by aphids. Entomologia Experimentalis Et Applicata, 24, 721-730.

Tjallingii, W. F. (1995). Regulation of phloem sap feeding by aphids. Regulatory mechanisms in insect feeding. New York: Chapman and Hall.

Tjallingii, W. F. (2006). Salivary secretions by aphids interacting with proteins of phloem wound responses. Journal of Experimental Botany, 57, 739-745.

Tjallingii, W. F., \& Esch, T. H. (1993). Fine-structure of aphid stylet routes in plant-tissues in correlation with EPG signals. Physiological Entomology, 18, 317-328.

van Emden, H. F., \& Harrington, R. (2007). Aphids as crop pests. London/Wallingford: CABI.

Vandermorten, S., Harmel, N., Mazzucchelli, G., De Pauw, E., Haubrage, E., \& Francis, F. (2014). Comparative analysis of salivary proteins from three aphid species. Insect Molecular Biology, $23,67-77$. 
Will, T., \& van Bel, A. J. (2006). Physical and chemical interactions between aphids and plants. Journal of Experimental Botany, 57, 729-737.

Will, T., Tjallingii, W. F., Thonnessen, A., \& van Bel, A. J. (2007). Molecular sabotage of plant defense by aphid saliva. Proceedings of the National Academy of Sciences of the United States of America, 104, 10536-10541.

Will, T., Carolan, J. C., \& Wilkinson, T. L. (2012). Breaching the sieve element - The role of saliva as the molecular interface between aphids and the phloem. In G. A. Thompson \& A. J. E. van Bel (Eds.), Phloem: molecular cell biology, systemic communication, biotic interactions. London: Wiley. 